\title{
MRI image segmentation using machine learning networks and level set approaches
}

\author{
Layth Kamil Adday Almajmaie', Ahmed Raad Raheem², Wisam Ali Mahmood ${ }^{3}$, Saad Albawi ${ }^{4}$ \\ ${ }^{1}$ Department of Computer Engineering, University of Technology-Iraq, Baghdad, Iraq \\ ${ }^{2}$ Department of Computer Science, Diyala Education Directorate, Baghdad, Iraq \\ ${ }^{3}$ Department of Computer Science, University of Technology-Iraq, Baghdad, Iraq \\ ${ }^{4}$ Department of Computer Engineering, University of Diyala, Baqubah, Iraq
}

\begin{tabular}{l} 
Article Info \\
\hline Article history: \\
Received Oct 23, 2020 \\
Revised Aug 26, 2021 \\
Accepted Sep 9, 2021 \\
\hline
\end{tabular}

Keywords:

Brain tumour

Fully convolutional network

Image segmentation

Level set

Seg-Net

U-Net

\begin{abstract}
The segmented brain tissues from magnetic resonance images (MRI) always pose substantive challenges to the clinical researcher community, especially while making precise estimation of such tissues. In the recent years, advancements in deep learning techniques, more specifically in fully convolution neural networks (FCN) have yielded path breaking results in segmenting brain tumour tissues with pin-point accuracy and precision, much to the relief of clinical physicians and researchers alike. A new hybrid deep learning architecture combining SegNet and U-Net techniques to segment brain tissue is proposed here. Here, a skip connection of the concerned U-Net network was suitably explored. The results indicated optimal multi-scale information generated from the SegNet, which was further exploited to obtain precise tissue boundaries from the brain images. Further, in order to ensure that the segmentation method performed better in conjunction with precisely delineated contours, the output is incorporated as the level set layer in the deep learning network. The proposed method primarily focused on analysing brain tumor segmentation (BraTS) 2017 and BraTS 2018, dedicated datasets dealing with MRI brain tumour. The results clearly indicate better performance in segmenting brain tumours than existing ones.
\end{abstract}

This is an open access article under the CC BY-SA license.

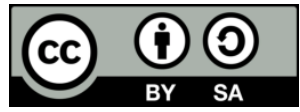

Corresponding Author:

Saad Albawi

Department of Computer Engineering, University of Diyala

Baqubah, Iraq

Email: saadalbawi@uodiyala.edu.iq

\section{INTRODUCTION}

While performing neurological classification of Parkinson's and Alzheimer's disease, clinical physicians primarily rely upon magnetic resonance images (MRI) for effective segmentation of brain tissues. But, while classifying MRIs of the brain tissues, the clinical researchers have to invariably struggle with certain inhibiting factors like low contrast, non-uniformity, and complex structure, right from the stage in which an image is acquired. However, in a much-needed relief, in a majority of cases now machine learning is being applied as a technique while performing MRI segmentation [1]. In many landmark cases, deep learning (DL) methods have successfully demonstrated application of these convolutional neural network (CNN) based techniques in the realm of brain tissue segmentation. In essence, three layers viz., the fully connected layer, pooling as well as convolution layer form primary building blocks of a regular CNN framework.

Moreover, the experience of earlier researchers has shown that it is a daunting and exhaustive task to recognize image paths in a typical CNN. The fully convolutional network (FCN), an end-to-end segmentation method has been introduced which makes a pixel-by-pixel prediction to produce the labels directly through analysis of images. In fact, on account of its better capacity to represent features, it produces better results in 
object detection, classification, and segmentation, though with availability of sufficient training data. In recent times, the FCN network has been extended through more recycling extra feature maps to generate promising results for U-net. It has done so with even smaller amounts of training samples. However, its computational inefficiency is a cause of concern. The brain images are effectively segmented using a computational DL technique capable of performing even on scanty training data. SegNet and U-Net are combined as hybrid architecture. A highly performing multi-scale information is generated using the SegNet architecture as the base, while skip connection is used at the select de-convolution layer. The model yields faster convergence when pooling indices pass into de-convolution layers. Then, counters of brain tissue boundaries are extracted by combining additional level set layer within this architecture. Final validation is done through real brain tumour data set. Section 2 reviews the relevant literature, while suggested method is presented and discussed in detail in section 3 . The final part 4 deals with presentation of the experimental results, which is discussed in detail as well as the conclusion.

\section{RELATED WORK}

Machine learning methods like the clustering and segmentation methods yield better results in tumour segmentation [1]-[3] used random forests (RF) to retrieve statistical features that are passed as input. In 2015, the best performer in the 2015 brain tumor segmentation (BraTS) challenge pioneered the application of convolutional neural networks to segment brain tumour images [4]. Some local features and global features are explored at the same time in this $\mathrm{CNN}$ architecture, clocking 30 times more speed than leading-edge solutions of the time. In addition, the convolutional implementation of a fully connected layer is applied in this architecture thus attaining 40 times acceleration. A CNN 3D architecture extracting patches of 3D voxels with varying brain MRI modalities [5]. 3D voxels are fed into a 4-layered CNN architecture to predict the tissue label of the centre voxel. More computational demands are prevented in 3D voxels by transforming 4D data into 2D data [6]. So that segmentation of brain tumours could be performed by 2D-CNN architecture. $11 \mathrm{CNN}$ Architecture layers are evaluated on the BraTS dataset in [7] when small filters (3x3) are fit into convolutional layers and comparative dice scores are reported. In alignment with classification or clustering methods, CNNs limit the problems in training data and improve performance [8]. An effective deep learning-based approach, known as DMRes, an improvised version of deep medic was developed for segmenting brain tumour [9], [10]. The early contributors attained some degree of efficacy by combining Level set evolution and global smoothness with flexible topology changes and mathematical morphology [11]. It gave them certain scoring points over traditional statistical classification. The method evaluated the working of the algorithm based on volume overlap and Hausdorff distance. While setting the equation parameters in speed function, level set algorithms face some difficulty. 3D tumour segmentation (TLS) was done by applying level sets through a threshold-based scheme [12]. The speed function is designed using a global threshold on the basis of confidence interval with iterative updates (search-based and adaptive) in which users are involved in varied degrees in the evolution process. A signed pressure function (SPF) with efficiency to block contours at weak or blurred edges was done [13]. The algorithm differentiates tumours from the rest of an image by using local statistics. Additionally, automatic calculation of image thresholding is done here.

Deep learning-based methods have been widely applied to many fields and have achieved state-ofthe-art performance. However, brain tumour segmentation poses several unique challenges. First, image quality has a critical impact on segmentation performance [14]. For example, blurred images result in poor outcomes. Second, image pre-processing steps also have an impact on the performance. For example, intensity normalization across cases is critical for tumour segmentation. Third, tumour tissue heterogeneity may pose a serious challenge to the developing an effective method. Finally, data imbalance is common and poses another intricate challenge for the use of deep learning [15].

\section{PROPOSED METHOD}

The proposed hybrid U-SegNet implementation as shown in the Figure 1. The input image is first entered into at the pre-processing stage and then trained by using hybrid CNN. Then, the level set extension (ELS) is integrated into Hybrid U-SegNet for extraction of boundaries from brain MRI images [16], [17].

\subsection{U-SegNet architecture}

U-SegNet architecture, a hybrid structure formed by using SegNet and U-Net architectures is projected in Figure 2. As local information scores over global information in separating white matter (WM), gray matter (GM), and cerebrospinal fluid (CSF), axial slices of size $256 * 128$ are assigned patch-based training. After observing the GM structures and noting the same in the chosen resolution dataset, local structures are formed on a patch size of 40 for segmentation. Input patches of $40 * 40 * 3$ are easily handled by the SegNet 
architecture at its best. A $3 \times 3$ kernel is used in each convolutional layer within the architecture in alignment with max-pool layers of size $2 * 2$, besides activating rectified linear unit (ReLU) activation functions. A skip connection of U-Net type is inserted just at the upper layer for clearly displaying characteristic maps as shown in Figure 2. Here, coarser and finer information is consolidated by using a 1x1 convolutional layer for segmenting purpose, besides transferring lesser parameters to the last convolutional layer. Similar to U-Net, fine information is incorporated without increasing the parameters through the skip connection. In the end, the 4-label classification as background (0), GM (1), WM (2) and CSF (3) is implemented by applying a SoftMax layer with 4 outputs as shown in Figure 2.

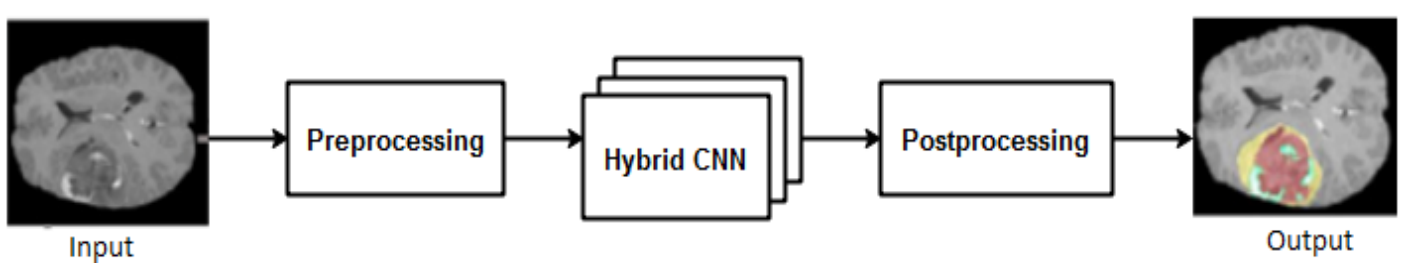

Figure 1. Block diagram of the purposed method

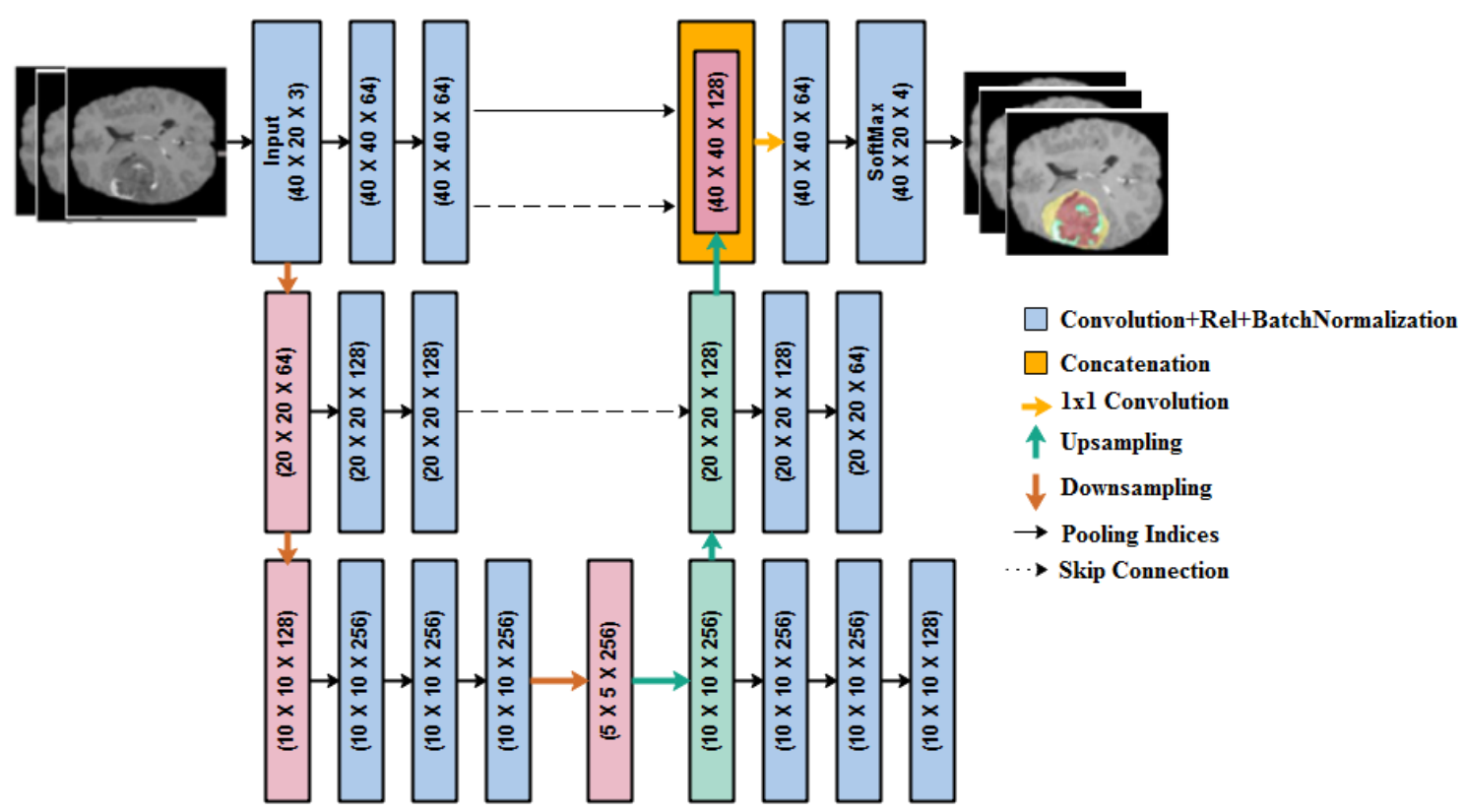

Figure 2. Fully convolutional network (FCN)

\subsection{Level set}

Image segmentation [18] makes wide application of level set (LS) method with active contouring (AC) as it performs automatic manoeuvring of possible topological changes. The potential of LS in attaining accuracy in segmenting brain tumour is documented in [12], [13].

\subsubsection{Background}

Consider the problem of segmentation of binary images in the dimension of $2 \mathrm{D}$ and to be denoted as $\Omega$. Next, also consider $C$ will be the boundary of desired open set and it to be defined as $C=\partial \mathrm{W}$, where $\mathrm{W} \in \Omega$. Now, [19] in case of the concept of LS framework the open set border to be defined as $C$ to be defined with $\phi$ the level zero game and is represented as shown in (1):

$$
\forall(X, Y)=\Omega\left\{\begin{array}{l}
C=\{(X, Y): \phi(X, Y)=0\} \\
\operatorname{in}(C)=\{(X, Y): \phi(X, Y)>0\} \\
\operatorname{out}(C)=\{(X, Y): \phi(X, Y)<0\}
\end{array}\right\}
$$


The segmentation task in which $\Omega$ to be represented as complete domain corresponds to I original image. Next, LS function to be denoted as $\phi$ and it grouped region $\Omega$ into two regions: inside region of W to be in $(\mathrm{C})$ and outside $\mathrm{W}$ to be defined as out(C). The length of the outline $\mathrm{C}$ is defined as shown in (2):

$$
\operatorname{Length}(C)=\int_{\Omega}|\nabla H(\phi(X, Y))| d x d y=\int_{\Omega} \delta(\phi(X, Y))|\nabla \phi(X, Y)| d x d y
$$

and the area inside the contour $\mathrm{C}$ is defined in

$$
\operatorname{Area}(C)=\int_{\Omega} H(\phi(X, Y)) d x d y
$$

Typically, in a LS-based segmentation method, the beginning us made with level set $\phi_{0}$ for the input image $I$. The through gradient descent is applied to initialize the update process in LS wherein an energy function that is a function of the variation in image attributes like colour and texture between the foreground and the background is minimized. The LS requires form and regions to improve performance. Since LS uses only lowlevel features, it is limited to reading complex images [20], [21]. However, deep networks are adept at learning and encoding critical features, thus helping in overcoming this limitation.

\subsubsection{Convolution layer}

In case of the convolution layer both the input and output to be represented as feature map. However, it is noticed that convolution output feature map is evaluated by performing the operation convolution with the feature map corresponds to the input layer and it is defined in (4).

$$
Y_{(S, \theta)}=f_{S}(X, \theta)=X * W_{S}+b
$$

From the (4) $X$ is to be considered as the convolution input layer feature map and $b$ noticed as the bias of the convolution kernel. Similarly, $W_{S}$ represented as the convolution at a stride and it to be represented as $s$. Finally, the output of the convolution layer feature map to be defined as $Y_{(S, \theta)}$ and is formed with the convolutional layers of stride $S$ and parameter $\theta$ and the result is down sampled.

\subsubsection{Deconvolution layer}

The input feature maps are oversampled by deconvolutional layer when maximum pooling indices from the related convolutional feature map is applied. The output of the deconvolutional feature map is concatenated with the matching convolutional feature map through a skip connection. The feature maps in a deconvolutional layer are used as output $Y_{(S, \theta)}$ picked from the preceding convolutional layer as its matching feature map. Let $G_{S}(; \tau)$ denotes a deconvolutional layer parameterized by $\tau$ which the convolution layer input with the factor and to be represented as $s$. Now, the output is resulted by concatenating relevant Convolutional Layer and is denoted as $Y_{(S-1, \theta)}$ using the skip connection and is defined as (5).

$$
\hat{Y}_{(s, \tau)}=\operatorname{concat}\left[G_{S}\left(Y_{(S, \theta)} ; \tau\right), Y_{(S-1, \theta)}\right]
$$

\subsubsection{Hybrid U-SegNet with level set layer}

Inspired from the existing frameworks mentioned as U-SegNet and the LS framework, proposed hybrid method combined with above two methods and the output feature maps are represented as $(Y)$ and is derived from $U-S e g N e t$ using the euclidean distance transformation $(\xi)$. The desired level set function to be represented in the (6).

$$
\phi=\xi(Y)-\xi(1-Y)
$$

From (6) the input space is represented as $\phi$. In order to minimize the energy function in the network model as shown in (7) is used and is defined as:

$$
\begin{aligned}
E\left(C_{1}, C_{2}, \phi\right)=\int_{\Omega} & \mu H(\phi)+\mu \delta(\phi)|\nabla \phi|+\alpha(H(\phi)-G T)^{2} \\
& +\lambda_{1}\left|H(\phi)-C_{1}\right|^{2} H(\phi)+\lambda_{2}\left|H(\phi)-C_{2}\right|^{2}(1 \\
& -H(\phi)) d x d y
\end{aligned}
$$


From the (7), it is observed that initial part denotes the inner contour and second part defied as the length of the contour $C$. Moreover, it is noticed that first part to be neglected in case of the $\mu=0$. Also, with similar to standard VLS, $v>0$ and it is useful for the noise free. But the study introduced $v<0$ on case of the brain tumour. Next, the third part is represented as ground truth images and complete part is neglected in case of the $\alpha>0$. At final, the last two parts related the energy in and out contour $C$. In case of the brain tumour, in and out counters are defined as $\lambda_{1}$ and $\lambda_{2}$ and also these values to be always positive. Finally, the two constants $C_{1}$ and $C_{2}$ are specified in (8) and (9) and are used to optimize the energy function $\phi$ as shown in Figure 3.

$$
\begin{aligned}
C_{1} & =\frac{\int_{\Omega} H(\phi)(x, y) H(\phi) d x d y}{\int_{\Omega} H(\phi) d x d y} \\
C_{2} & =\frac{\int_{\Omega} H(\phi)(x, y)(1-H(\phi)) d x d y}{\int_{\Omega}(1-H(\phi)) d x d y}
\end{aligned}
$$

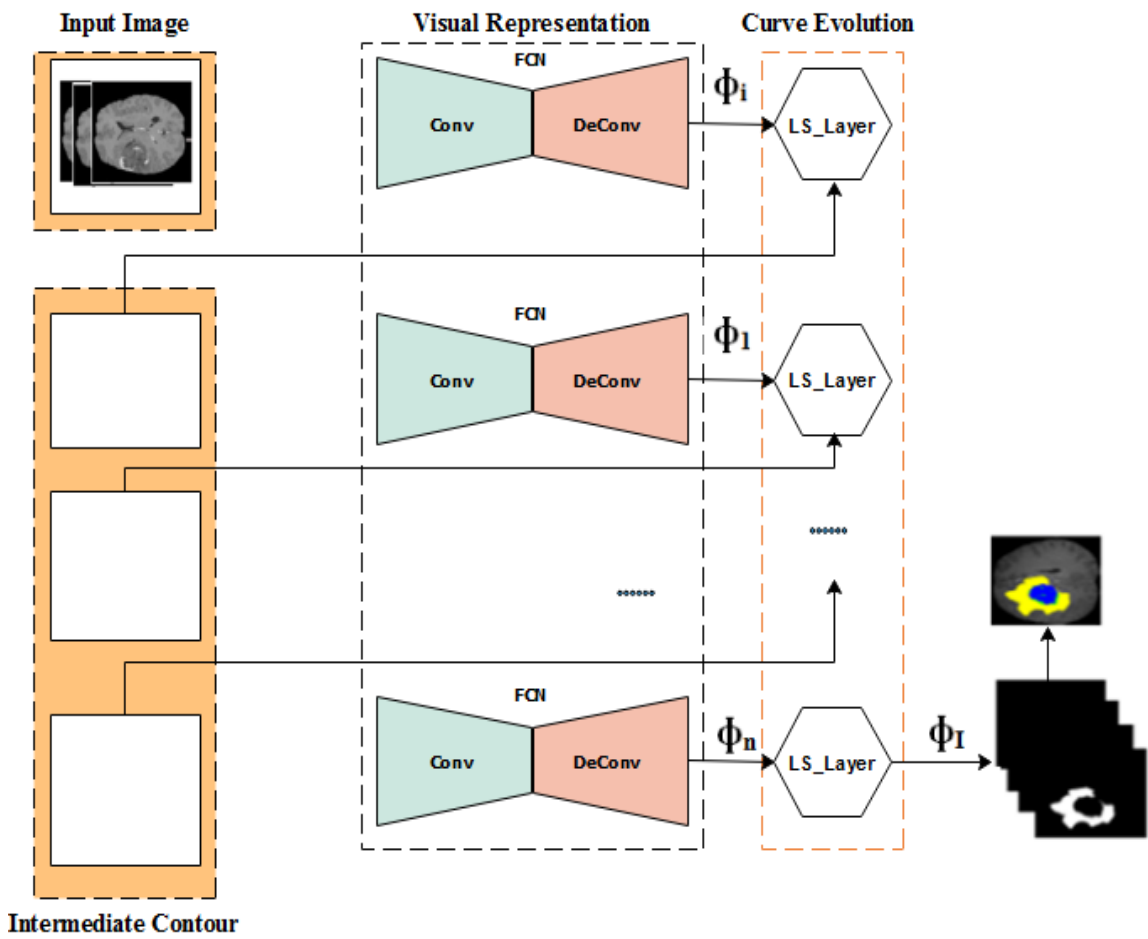

Figure 3. Proposed hybrid method

\section{EXPERIMENT RESULT}

\subsection{Dataset and measurement}

BraTS data forms the training data for the new diagnostic reference levels (DRLs) method. It also applies BraTS 2013 and BraTS 2015 datasets to prove its better show than standard techniques. Also, BraTS 2017 dataset is provided by medical image computing and computer-assisted intervention (MICCAI) for automated brain tumour segmentation task. Each dataset contains two subsets corresponding to low-grade glioma (LGG) and high grade gliomas (HGG) [22]. The dataset comprises training and testing datasets at $80 \%$ and $20 \%$, respectively. BraTS 2017 uses 168 HGG and 60 LGG training set to test this network that is later applied BraTS 2015 and 2013 datasets.

\subsection{Evaluation metrics}

The validation of this method is validated using metrics as detailed below:

\subsubsection{True positive}

'Sensitivity' or 'recall' represent true positives, denoting that detects the condition when such a condition is there [23]. The True positive rate measure is proportion of the relation of both true positive rate as well as the addition of false negative rate and true positive rate. 
$T P R=T P /(T P+F N)$

\subsubsection{True negative}

True negative is also termed as 'specificity'. When as fraction of true negative are added to true negative and false positive, we notice true negative. It denotes the test result that does not identify the condition when the condition is present.

$$
T N R=T N /(F P+T N)
$$

\subsubsection{False negative}

The proportion of false negative and the sum of true negative and false negative is referred to as false negative rate. It represents test result that detects the condition when the condition is absent.

$$
P p v=\mathrm{a} /(a+c)
$$

\subsubsection{False positive}

The fraction of false positive and the sum of false positive and true positive is added to get false positive rate. It indicates the test result that does not identify the condition when the condition is not present or absent.

$$
F P R=F P /(T P+F P)
$$

\subsubsection{Positive predictive value}

In case of a likelihood of a positive test when disease is present in the body of the patient, then positive predictive value is derived

$$
P p v=\mathrm{a} /(a+c)
$$

\subsubsection{Negative predictive value}

Negative predictive is defined as the possibility of no presence of the disease, which can be a harmful test.

$$
N P V=d / b+d
$$

\subsubsection{Dice similarity coefficient (DSC)}

Dice coefficient is one of the methods to establish the extent of the latitudinal connection between two binary images. The segmentation process is commonly used as the performance measures of dice coefficient, which provides more weighting to the instances [24], [25]. These values also range between zero and one. Dice coefficient is utilized to find the match between two similarities labelled as region (A and B) in the images, which is computed as:

$$
\begin{aligned}
& D=\frac{2(A G)}{A G+A G} \times 100 \\
& D S C=\frac{2 T P}{F P+2 T P+F N}
\end{aligned}
$$

The quantitative evaluation based on Sensitivity (SEN) and Specificity (SPE) is given by the equation:

$$
\begin{aligned}
& S E N=\frac{T P}{T P+F N} \\
& S P E=\frac{T N}{T N+F P}
\end{aligned}
$$

\subsection{Results and discussion}

The proposed algorithm achieved average dice scores of $0.89,0.79$, and 0.74 for whole tumour (WT), core tumour (CT) and enhancing CT regions, respectively in the HGG case on the 2013 BraTS dataset. In the case of LGG, the proposed model achieved dice scores of $0.89,0.62$, and 0.43 for WT, CT, and enhancing CT 
regions. Thus, it has achieved high performance, which is better than the other standard methods. The sensitivity and specificity values achieved on BraTS 2013 dataset are $0.90,0.89$, and 0.93 and $0.92,0.84$, and 0.86, respectively. Similarly, in case of LGG, the sensitivity and specificity values achieved on BraTS 2013 dataset are $0.89,0.83$, and 0.85 and $0.91,0.80$, and 0.84 , respectively.

Based on the 2015 BraTS dataset, WT, CT, and enhancing CT regions receive dice score, $0.82,0.73$ and 0.68 in HGG case. In case of LGG, for WT, CT, and enhancing CT regions receive dice scores of 0.82 , 0.57 , and 0.40 in this model. Thus, it has achieved high performance, which is better than the other standard methods. The sensitivity and specificity values achieved on BraTS 2013 dataset are $0.83,0.82$, and 0.86 ); and $0.85,0.77$, and 0.79 , respectively. Similarly, in case of LGG, the sensitivity and specificity values achieved on BraTS 2013 dataset are $0.82,0.76$, and 0.78 ; and $0.84,0.74$, and 0.77 , respectively.

As shown in Table 1 and Table 2, there are consistent better dice scores in sensitivity and specificity produced by the proposed algorithm. The performance can be credited to the availability of additional training data from both 2013 and 2015 BraTS dataset that helped in fine tuning the hyper-parameters of this algorithm. Such as reliability against outliers, speed makes this algorithm achieve better segmentation of core tumour. Figure 4 shows the result of proposed method, quantitatively and shows that the proposed method produces best segmentation results as compared to the standard U-Net method.

Table 1. Performance of proposed method vs standard methods tested via BraTS 2013 dataset

\begin{tabular}{ccccccccccc}
\hline & & \multicolumn{3}{c}{ Dice Score } & \multicolumn{3}{c}{ Sensitivity } & \multicolumn{3}{c}{ Specificity } \\
& & WT & CT & ET & WT & CT & ET & WT & CT & ET \\
\hline \multirow{2}{*}{ Pereira16 U-Net } & HGG & 0.88 & 0.76 & 0.73 & 0.87 & 0.79 & 0.80 & 0.89 & 0.79 & 0.68 \\
& LGG & 0.65 & 0.53 & 0.00 & 0.71 & 0.75 & 0.72 & 0.75 & 0.73 & 0.69 \\
Havaei16 U-Net & HGG & 0.88 & 0.79 & 0.73 & 0.62 & 0.68 & 0.72 & 0.82 & 0.78 & 0.80 \\
& LGG & 0.88 & 0.58 & 0.21 & 0.78 & 0.81 & 0.82 & 0.76 & 0.79 & 0.81 \\
Proposed & HGG & 0.89 & 0.79 & 0.74 & 0.90 & 0.89 & 0.93 & 0.92 & 0.84 & 0.86 \\
& LGG & 0.89 & 0.62 & 0.43 & 0.89 & 0.83 & 0.85 & 0.91 & 0.80 & 0.84 \\
\hline
\end{tabular}

Table 2. Performance variations in methods on BraTS 2015 dataset

\begin{tabular}{ccccccccccc}
\hline & & \multicolumn{3}{c}{ Dice Score } & \multicolumn{3}{c}{ Sensitivity } & \multicolumn{3}{c}{ Specificity } \\
& & WT & CT & ET & WT & CT & ET & WT & CT & ET \\
\hline \multirow{2}{*}{ Pereira16 U-Net } & HGG & 0.81 & 0.70 & 0.67 & 0.80 & 0.73 & 0.74 & 0.82 & 0.73 & 0.63 \\
& LGG & 0.60 & 0.49 & 0.00 & 0.65 & 0.69 & 0.66 & 0.69 & 0.67 & 0.63 \\
Havaei16 U-Net & HGG & 0.81 & 0.73 & 0.67 & 0.57 & 0.63 & 0.66 & 0.75 & 0.72 & 0.74 \\
& LGG & 0.81 & 0.53 & 0.19 & 0.72 & 0.75 & 0.75 & 0.70 & 0.73 & 0.75 \\
Proposed & HGG & 0.82 & 0.73 & 0.68 & 0.83 & 0.82 & 0.86 & 0.85 & 0.77 & 0.79 \\
& LGG & 0.82 & 0.57 & 0.40 & 0.82 & 0.76 & 0.78 & 0.84 & 0.74 & 0.77 \\
\hline
\end{tabular}
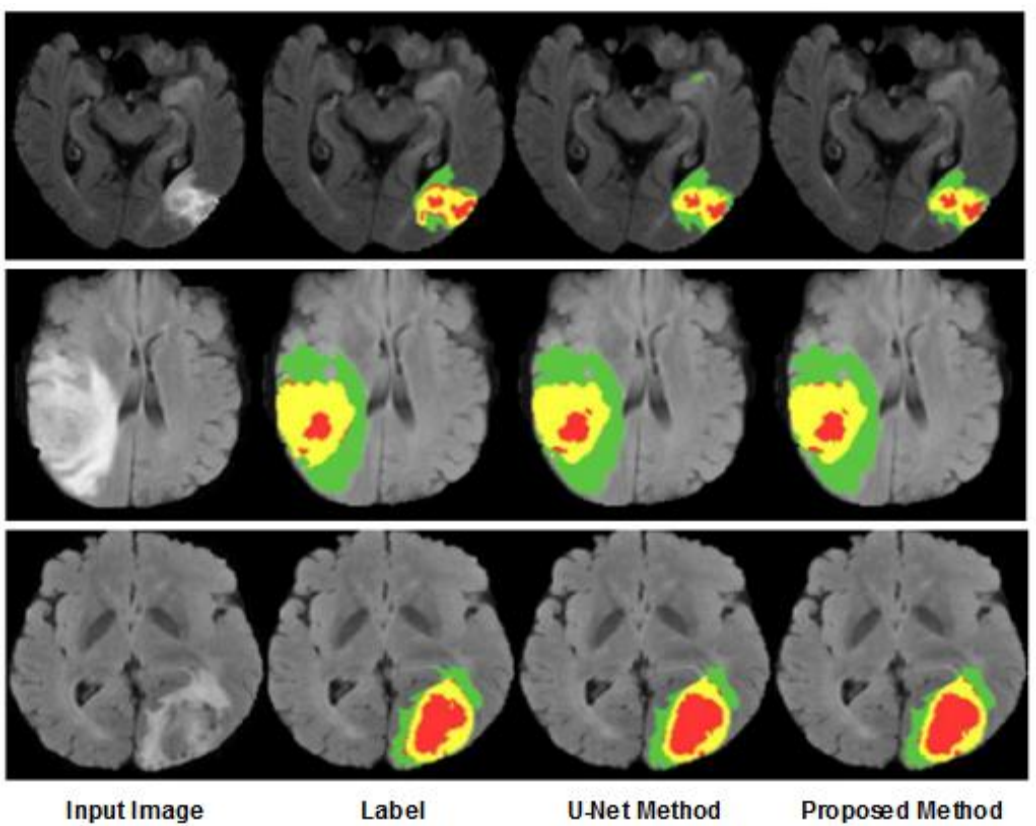

Figure 4. Comparison results of sample images from left to right, ground truth, U-Net, and proposed method 


\section{CONCLUSION}

This new algorithm makes use of deep recurrent level sets and integrates the advantages of both deep learning for carrying out brain tumour segmentation as an automatic process. The existing standard models have also been briefly discussed to achieve contextual orientation. The results obtained confirm that by integrating level sets and recurrent FCN architectures, the proposed DRLs offers superior solution through its robustness against outliers, speed and consistent while segmenting core tumour. Additionally, DRLs improves the speed of segmenting brain tumours to a large extent, thus making it a practical solution. Consequently, the results demonstrate that the proposed methods show state-of-the-art performance in all three tasks with sufficient robustness to handle data from multiple datasets. In future, we plan extensions to the proposed architecture by integrating whole slide image and molecular genetic features for tumour classification following new WHO criterion4.

\section{REFERENCES}

[1] V. V. Priya and Shobarani, "An efficient segmentation approach for brain tumor detection in MRI," Indian Journal of Science Technology, vol. 9, no. 19, pp. 1-6, 2016, doi: 10.17485/ijst/2016/v9i19/90440.

[2] M. Kopec, M. Błaszczyk, M. Radek, and H. Abramcsyk, "Raman imaging and statistical methods for analysis various type of human brain tumors and breast cancers," Spectrochimica Acta Part A: Molecular and Biomolecular Spectroscopy, vol. 262, Dec. 2021, Art. no. 120091, doi: 10.1016/j.saa.2021.120091.

[3] P. Afshar, K. N. Plataniotis, and A. Mohammadi, "Capsule networks for brain tumor classification based on mri images and coarse tumor boundaries, ” ICASSP 2019 - 2019 IEEE International Conference on Acoustics, Speech and Signal Processing (ICASSP), 2019, pp. 1368-1372, doi: 10.1109/ICASSP.2019.8683759.

[4] J. Byrne, R. Dwivedi, and D. Minks. "Recommendations for cross-sectional imaging in cancer management, Second edition, (Tumours of the brain)." The Royal College of Radiologists. https://www.rcr.ac.uk/publication/recommendations-cross-sectionalimaging-cancer-management-second-edition (accessed Nov. 5, 2019).

[5] R. R. Agravat and M. S. Raval, "Prediction of overall survival of brain tumor patients," TENCON 2019 - 2019 IEEE Region 10 Conference (TENCON), 2019, pp. 31-35, doi: 10.1109/TENCON.2019.8929497.

[6] P. Mlynarski, H. Delingette, A. Criminisi, and N. Ayache, "Deep learning with mixed supervision for brain tumor segmentation," Journal of Medical Imaging, vol. 6, no. 3, 2019, Art. no. 034002, doi: 10.1117/1.JMI.6.3.034002.

[7] J. Amin, M. Sharif, M. Yasmin, and S. L. Fernandes, "Big data analysis for brain tumor detection: Deep convolutional neural networks," Future Generation Computer Systems, vol. 87, pp. 290-297, 2018, doi: 10.1016/j.future.2018.04.065.

[8] K. Usman and K. Rajpoot, "Brain tumor classification from multi-modality MRI using wavelets and machine learning," Pattern Analysis and Application, vol. 20, pp. 871-881, 2017, doi: 10.1007/s10044-017-0597-8.

[9] S. Pereira, R. Meier, V. Alves, M. Reyes, and C. A. Silva, "Automatic brain tumor grading from MRI data using convolutional neural networks and quality assessment," in IMIMIC 2018: Understanding and Interpreting Machine Learning in Medical Image Computing Applications, 2018, vol. 11038, pp. 106-114, doi: 10.1007/978-3-030-02628-8_12.

[10] S. Vijih, S. Sharma, and P. Gaurav, "Brain tumor segmentation using OTSU embedded adaptive particle swarm optimization method and convolutional neural network," in Data Visualization and Knowledge Engineering, vol. 32, pp. 171-194, doi: 10.1007/978-3-030-25797-2_8.

[11] H. Mohsen, E.-S. A. El-Dahshan, E.-S. M. El-Horbaty, and A. B. M. Salem, "Classification using deep learning neural networks for brain tumors," Future Computing and Informatics Journal, vol. 3, no. 1, pp. 68-71, 2018, doi: 10.1016/j.fcij.2017.12.001.

[12] J. Cheng, 2017, "Brain Tumor Dataset," Figshare, doi: 10.6084/m9.figshare.1512427.v5.

[13] M. Sajjad, S. Khan, K. Muhammad, W. Wu, A. Ullah, and S. W. Baik, "Multi-grade brain tumor classification using deep CNN with extensive data augmentation," Journal of Computational Science, vol. 30, pp. 174-182, 2019, doi: 10.1016/j.jocs.2018.12.003.

[14] T. T. Wong, "Performance evaluation of classification algorithms by k-fold and leave-one-out cross validation," Pattern Recognitiction, vol. 48, no. 9, pp. 2839-2846, 2015, doi: 10.1016/j.patcog.2015.03.009.

[15] S. Hussain, S. M. Anwar, and M. Majid, "Segmentation of glioma tumors in brain using deep convolutional neural network," Neurocomputing, vol. 282, pp. 248-261, 2018, doi: 10.1016/j.neucom.2017.12.032.

[16] K. G. Khambhata and S. R. Panchal, "Multiclass classification of brain tumor in MR images," International Journal of Innovative Research in Computer and Communication Engineering, vol. 4, no. 5, pp. 8982-8992, May 2016.

[17] S. Koley, A. K. Sadhu, P. Mitra, B. Chakraborty, and C. Chakraborty, "Delineation and diagnosis of brain tumors from post contrast T1-weighted MR images using rough granular computing and random forest," Applied Soft Computing, vol. 41, pp. 453-465, 2016, doi: 10.1016/j.asoc.2016.01.022.

[18] U. Ilhan and A. Ilhan, "Brain tumor segmentation based on a new threshold approach," Procedia Computer Science, vol. 120, pp. 580-587, 2017, doi: 10.1016/j.procs.2017.11.282.

[19] Z. Ji, Y. Huang, Q. Sun, and G. Cao, "A spatially constrained generative asymmetric Gaussian mixture model for image segmentation," Journal of Visual Communication and Image Representation, vol. pp. 611-626, 2016, doi: 10.1016/j.jvcir.2016.08.001.

[20] A. Kamboj, R. Rani, and J. Chaudhary, "Deep leaming approaches for brain tumor segmentation: A review," 2018 First International Conference on Secure Cyber Computing and Communication (ICSCCC), 2018, pp. 599-603, doi: 10.1109/ICSCCC.2018.8703202.

[21] S. Saeb, L. Lonini, A. Jayaraman, D. C. Mohr, and K. P. Kording, "The need to approximate the use-case in clinical machine learning," Gigascience, vol. 6, no. 4, pp. 1-9, 2017, doi: 10.1093/gigascience/gix019.

[22] M. Havaei et al., "Brain tumor segmentation with deep neural networks," Medical Image Analysis, vol. 35, pp. 18-31, 2017, doi: 10.1016/j.media.2016.05.004.

[23] S. Hussain, S. M. Anwar and M. Majid, "Brain tumor segmentation using cascaded deep convolutional neural network," 2017 39th Annual International Conference of the IEEE Engineering in Medicine and Biology Society (EMBC), 2017, pp. 1998-2001, doi: 10.1109/EMBC.2017.8037243.

[24] S. Iqbal, M. U. Ghani, T. Saba, and A. Rehman, "Brain tumor segmentation in multi- spectral MRI using convolutional neural networks (CNN)," Microscopy Research and Technique, vol. 81, no. 4, pp. 419-427, 2018, doi: 10.1002/jemt.22994. 
[25] S. Pereira, A. Pinto, V. Alves, and C. A. Silva, "Brain tumor segmentation using convolutional neural networks in MRI images," in IEEE Transactions on Medical Imaging, vol. 35, no. 5, pp. 1240-1251, May 2016, doi: 10.1109/TMI.2016.2538465.

\section{BIOGRAPHIES OF AUTHORS}

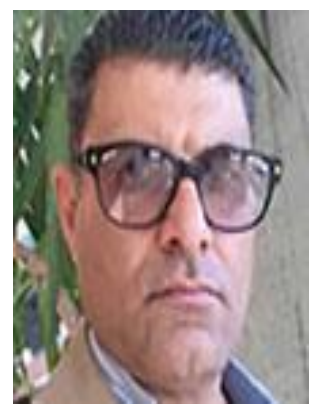

Layth kamil Adday Almajmaie (iD SOS SC P is a faculty member at the University of Technology, Department of Computer Engineering for more than 14 years, He was awarded a master degree from University of SAM India, in 2012, and Ph.D. from Department of Computer Engineering, University of Ultinbas, Turkey. His research interest is in network, wireless network, network security, data mining-cloud computing, distributed database, and image processing. He can be contacted at email: 10884@uotechnology.edu.iq.

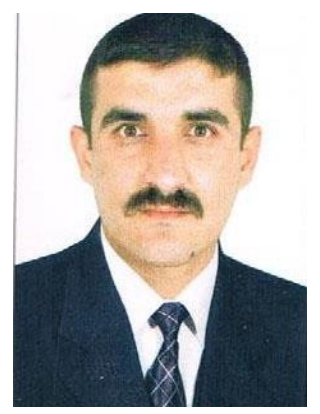

Ahmed Raad Raheem (DD SA SC P received his B.Sc. from University of Diyala, Iraq 20032007 and M.Sc. from Sam Higginbottom University of Agriculture, Technology and Sciences, India 2010-2012, currently he is lecturer in Diyala Education Directorate-Ministry of Education. He can be contacted at email: Ahmedraadr@yahoo.com.

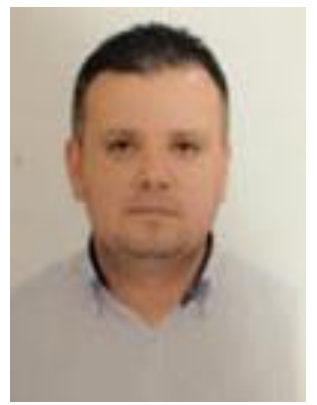

Wisam Ali Mahmood (D) SC SC is a faculty member at the University of Technology, Department of computer science for more than 11 years, He was awarded a master degree from University of SAM India, in 2012, His research interest is in network, wireless network, network security, data mining, cloud computing, distributed database, image processing. He can be contacted at email: 10860@uotechnology.edu.iq.

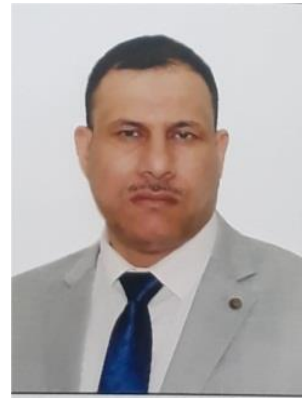

Saad Albawi (iD 8I SC P is a faculty member at the University of Diyala, Iraq, Department of Computer Engineering, University of Technology-Iraq, Ph.D. from Department of Computer Engineering, University of Ultinbas, Turky, and Work as a lecturer at University of Diyala, Iraq. He can be contacted at email: saadalbawi@uodiyala.edu.iq. 